\title{
Movilidad y desarrollo translocal en la Nicaragua (semi-)rural ${ }^{1}$
}

\author{
Griet Steel, NannekeWinters ${ }^{* *}$ y Carlos Sosa ${ }^{* * *}$
}

Recibido: mayo de 2012 / Aceptado: junio de 2012

Este artículo pretende contribuir al debate sobre los vínculos entre la movilidad y el desarrollo, explorando el concepto de desarrollo translocal. Basado en trabajo de campo en los municipios de Matiguás y Muy Muy, éste analiza cómo la movilidad da forma a las estrategias de vida de los hogares (semi-)rurales en Nicaragua, y explora cómo los diferentes miembros de un hogar utilizan la movilidad física como una estrategia de vida. Argumenta que los habitantes de áreas (semi-)rurales consideran distintos tipos de movimientos como estrategias importantes para establecer enlaces entre personas y lugares, y para alcanzar un mejor bienestar en su comunidad natal. Al mismo tiempo muestra cómo la movilidad se forma en una arena de poder, lo que afecta su potencial. De esta manera, este artículo contribuye a un entendimiento dinámico y multidimensional de cómo los procesos de desarrollo dan forma a - y son formados por - la movilidad y la interconectividad.

Palabras clave: desarrollo translocal / hogares (semi-) rurales / estrategias de vida / movilidad / Nicaragua

1 Este artículo apareció originalmente en Inglés en una edición especial de "International Development Planning Review" (IDPR) sobre Desarrollo Translocal, Corredores de Desarrollo y Cadenas de Desarrollo, bajo la referencia: Steel, G., Winters, N. Eु Sosa, C. (2011). Mobility, translocal development and the shaping of development corridors in (semi-)rural Nicaragua. IDPR, 33 (4), 409-428.

* Antropóloga, investigadora posdoctoral en el Instituto de Políticas y Gestión del Desarrollo (IOB), Universidad de Amberes, Bélgica. Correo electrónico: griet.steel@ua.ac.be.

** Investigadora de doctorado en el Instituto de Políticas y Gestión del Desarrollo (IOB), Universidad de Amberes, Bélgica. Correo electrónico: nanneke.winters@ua.ac.be

*** Sociólogo, estudiante del Master en Globalización y Desarrollo en el Instituto de Políticas y Gestión del Desarrollo, IOB, Amberes, Bélgica (anteriormente investigador en el Instituto de Investigación para el Desarrollo de la Universidad Centroamericana, NITLAPAN-UCA).Correo electrónico: soscarlos@gmail. com 


\section{Introducción}

En este mundo globalizado las personas y las localidades se conectan cada vez más como resultado de lo que Massey (1991, p. 4) llama, "la fragmentación geográfica y la disrupción espacial de nuestro tiempo". Como consecuencia de este aumento en la interconectividad entre personas y lugares, y, en línea con las investigaciones contemporáneas sobre la migración internacional, la noción de movilidad ha ganado un creciente interés entre los investigadores de varias disciplinas (Cresswell, 2006; De Haas \& Rodríguez, 2010; Sheller \& Urry, 2006; Willis, 2010). El "new mobilities paradigm" o nuevo paradigma de la movilidad ha permitido a los científicos sociales desarrollar "una ciencia social impulsada por el movimiento, en la cual el movimiento, el movimiento potencial y el movimiento bloqueado están todos conceptualizados como una construcción de relaciones económicas, sociales y políticas" (Urry, 2007, p. 43). Este enfoque de movilidad ha coincidido con un creciente número de estudios sobre la multi-localidad de estrategias de vida (Brandao \& Zoomers, 2010; De Haan \& Zoomers, 2003; Paerregaard, 1998; Steel \& Zoomers, 2009; Thieme, 2008). Estos estudios reconocen la significancia de mover y construir lazos entre diferentes localidades para poder beneficiarse de las oportunidades en distintas localidades y por tanto mejorar los medios de vida (Ellis, 2000). La gente tiende a extender sus estrategias de vida a lo largo de lo local, regional, nacional e internacional (Sørensen \& Olwig, 2002). Se cree que estos medios de vida multilocales no sólo resultan en una expansión de oportunidades económicas, sino que también pueden incrementar las conexiones sociales, culturales y políticas entre áreas geográficamente distantes (Duany, 2002). Además, la movilidad es un aspecto recurrente en discursos populares sobre el alivio de la pobreza. En muchos países latinoamericanos, las expresiones locales de bienestar están explícitamente relacionadas a ideas de movimiento. Por ejemplo, la frase "seguir adelante" es comúnmente utilizada para expresar el bienestar mejorado y, por tanto, refleja la creencia popular de que la movilidad es un medio importante para escapar de la pobreza (Leinaweaver, 2008).

Sin embargo, como la movilidad está unida al desarrollo local, es sujeto de un continuo debate y requiere más estudio. Investigaciones sobre el nexo migración-desarrollo han dejado en claro que no existe un vínculo inequívoco, positivo o negativo, entre la movilidad de las personas y el desarrollo (De Haas, 2005). Por tanto, el debate debe ser enriquecido con casos concretos que analicen el nexo en escenarios históricos y locales específicos (De Haas \& Rodríguez, 2010). Adicionalmente, se debe considerar una noción de desarrollo más dinámica y tomar en cuenta la diversidad de los flujos de personas entre diferentes lugares.

Esta contribución se suma a ese cuerpo de conocimiento al aplicar el concepto de "desarrollo translocal", que enfatiza cómo las múltiples pertenencias de personas a distintas localidades influencian el proceso de desarrollo en una localidad específica (ver Zoomers \& Van Westen, 2011; ver también Duany, 2002). La propuesta considera estos procesos de desarrollo primeramente en términos de consumo, producción y empleo. Se enfoca en los hogares (semi-)rurales de Nicaragua para analizar cómo la movilidad y los enlaces entre personas y lugares dan forma a sus estrategias de vida. Este artículo explora cómo los diferentes miembros de estos 
hogares usan el movimiento como una estrategia para alcanzar un mejor bienestar en su comunidad natal. Al mismo tiempo, provee una percepción sobre cómo el movimiento físico, al igual que cualquier otro activo, es formado en una arena de poder que afecta su potencial. Este enfoque nos permite alejarnos de nociones estáticas y simplistas sobre la migración y el desarrollo para acercarnos a una conceptualización más dinámica, multidimensional y aterrizada en lo empírico. De este modo pretendemos contribuir a un mejor entendimiento de cómo los procesos de desarrollo dan forma - y son formados por - la movilidad y la interconectividad.

Este artículo está basado en cuatro meses de trabajo de campo etnográfico realizados en 2009-2010 en Matiguás y Muy Muy, dos municipios del departamento de Matagalpa en la región central de Nicaragua (ver ilustración 1) que totalizan alrededor de 56,000 habitantes, un cuarto de los cuales vive en el pueblo de Muy Muy, mientras que el resto vive en las comarcas. Los hogares que forman parte de nuestra investigación están localizados tanto en las comarcas como en el pueblo de Muy Muy². Casi todos mantienen lazos con el área rural y con el poblado. Por tanto, nombramos a estos hogares como semi-rurales ${ }^{3}$. Nuestro equipo de tres investigadores utilizó una triangulación de métodos de investigación cualitativos para obtener un mejor entendimiento de las estrategias de vida y la movilidad de las personas. Entrevistamos veinticinco hombres y cuarenta y cinco mujeres pertenecientes a cuarenta y seis hogares distintos, la mayoría en más de una ocasión. Aunque estos hogares son predominantemente jóvenes (la mayoría parejas jóvenes de entre 20 y 30 años, con niños pequeños), una porción significativa incluye miembros mayores, usualmente abuelas. Adicionalmente, utilizamos la observación participativa y experimentamos con talleres creativos, métodos visuales como mapeo social y foto voz (Collier \& Collier, 1996) así como diarios financieros (Collins, Mordoch, Rutherford \& Ruthven, 2009), con el propósito de permitir que los informantes se expresaran fuera del formato convencional de preguntas y respuestas.

2 En el municipio de Muy Muy llevamos a cabo la investigación en la ciudad misma así como en las comarcas El Bálsamo 1, El Bálsamo 2, El Dorado y La Luna. En el municipio de Matiguás llevamos a cabo investigaciones en las comarcas El Jobo, Pancasán y Sitio Histórico.

3 Seguimos a Ellis (2000 p. 18) quien define al hogar como un lugar de "intensa interdependencia social y económica" que "representa una coalición de actores, comprometidos por elección propia o costumbre, a actuar como una unidad de cara al resto del mundo". Esta definición no excluye la posibilidad para un hogar de ser espacialmente extendido ni los conflictos que conlleva (Ellis, 2000, p. 19), dos caracteristicas importantes en el contexto de investigaciones sobre movilidad. 


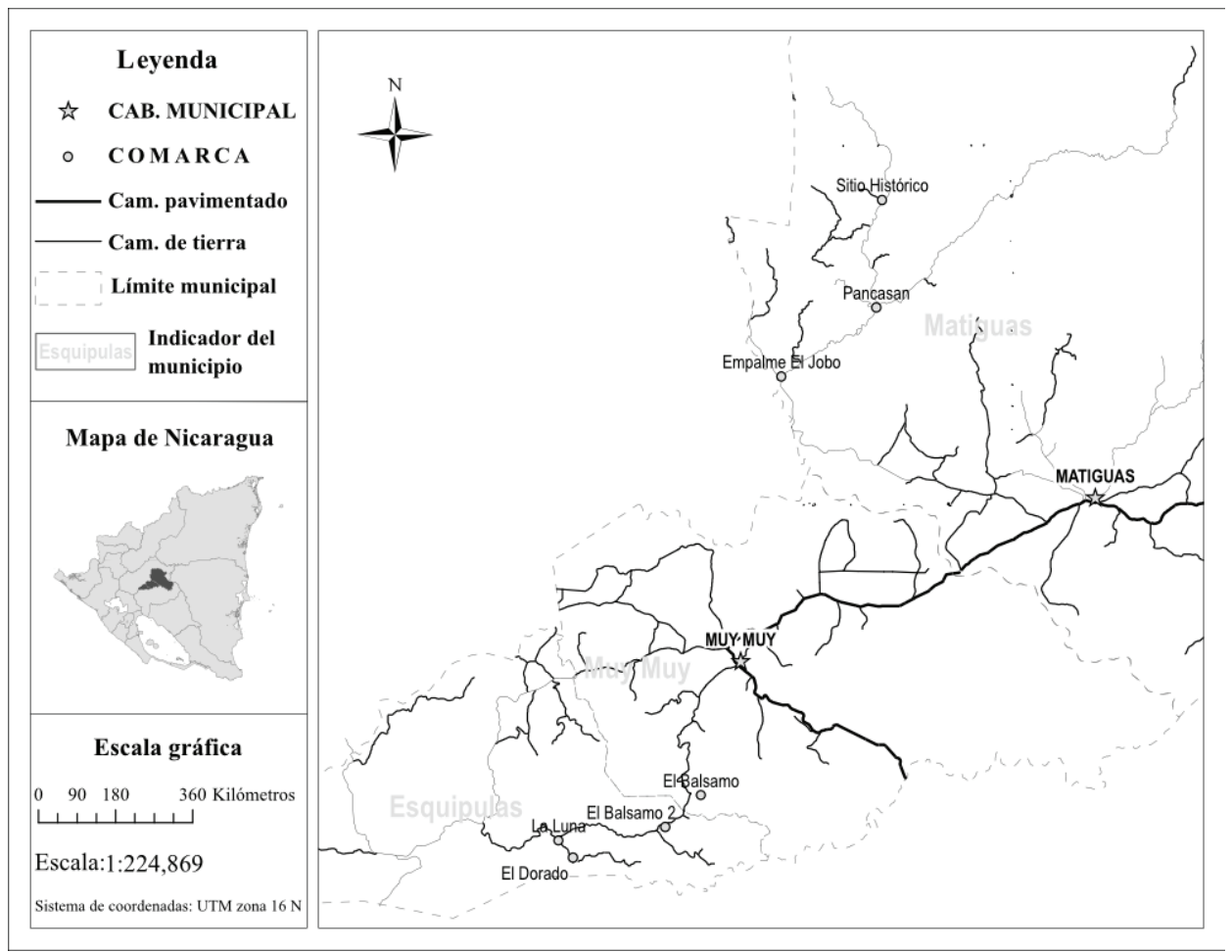

Ilustración 1. Área de investigación en Matiguás/Muy Muy, Nicaragua (semi-)rural Fuente: Carlos Sosa, Instituto de Investigación y Desarrollo, Nitlapan, 2011

Primero proveemos algunos antecedentes teóricos sobre la migración, la translocalidad y la apropiación del paradigma de la movilidad en estudios sobre desarrollo y estrategias de vida. En la sección empírica introducimos el área de la investigación, enfocándonos en la falta de oportunidades económicas viables en la región y cómo esto induce a los habitantes a moverse lejos de la comunidad. Sobre esta base distinguimos tres grupos que experimentan la movilidad de forma distinta: los hogares jóvenes, las madres solteras y las parejas. En la sección final analizamos cómo los varios actores establecen conexiones importantes entre los diferentes lugares y personas, y la importancia de estos enlaces para un mejor bienestar. En la conclusión el punto principal de nuestro argumento es puesto en la perspectiva más amplia del debate sobre la importancia de la movilidad y el desarrollo translocal en los corredores de desarrollo (Zoomers \& Van Westen, 2011).

\section{Migración, translocalidad y movilidad en las estrategias de vida}

Por varias décadas los estudios sobre migración han analizado la relación entre el desarrollo y la creciente interconectividad o la expansión espacial de las relaciones socioeconómicas. El Informe sobre Desarrollo Humano, SuperandoBarreras: Movilidad Humana y Desarrollo (PNUD, 2009) enfatiza la importancia de la movilidad humana 
en el proceso de desarrollo al afirmar que la migración es efectiva para aumentar el ingreso, la educación y la participación de individuos y familias, así como para mejorar su futuro. Sin embargo, dentro de las ciencias sociales, las implicaciones de la búsqueda de una mejor vida a través de la migración - el llamado nexo migracióndesarrollo - son aún sujeto de debate (De Haan, 1999; 2006). No obstante este debate generalmente es obstaculizado por una noción simplista y estática tanto de la migración como del desarrollo, que tiende a omitir varias dimensiones interrelacionadas que son parte constituyente de estos fenómenos. Por ejemplo, los estudios sobre el nexo migración-desarrollo tienden a adoptar un enfoque "determinado por el lugar" y dejan de lado las dinámicas y complejidades que caracterizan los procesos de migración (Schapendonk, 2011). A menudo descuidan las relaciones de poder que afectan el potencial de desarrollo de la migración. Analizan el desarrollo local desde una perspectiva cerrada y sedentaria que encuentra el desarrollo fijado en el tiempo y en el espacio (Zoomers \& Van Westen, 2011). Además, en estos estudios la migración es usualmente presentada o como un obstáculo o como una solución al desarrollo, en lugar de presentarla como "una parte integral" del mismo (De Haas \& Rodríguez, 2010, p. 179). Por tanto, fallan al integrar el proceso estructural y la mediación humana para permitir una interpretación más realista de la migración y el desarrollo (De Haas \& Rodríguez, 2010, pp. 178-1800).

Un creciente reconocimiento de la naturaleza dinámica y multi-dimensional de la migración y el desarrollo, así como un enfoque más claro sobre los múltiples enlaces entre personas y lugares, permite un análisis más productivo del nexo migración-desarrollo. La perspectiva transnacional en particular, ha jugado un importante papel en nuestro entendimiento de estos enlaces. El transnacionalismo, inicialmente definido como "el proceso mediante el cual los migrantes construyen espacios sociales que unen a su país de origen y al país en que se establecieron" (Glick Schiller, Basch \& Blanc-Szanton, 1992, pp. 26-27), ha proveído un punto de inicio interesante para analizar las conexiones de los migrantes con distintos países. Ha estimulado un enfoque en las estrategias de vida espacialmente dispersas, así como el uso activo de las escalas geográficas como un recurso por parte de los migrantes (Bebbington \& Battterbury, 2001, p. 374). El transnacionalismo, por tanto, ha introducido un nuevo enfoque interdisciplinario para "el pensamiento sobre las conexiones entre el 'aquí' y el 'allá”' (Waldinger \& Fitzgerald, 2004, pp. 1177).

A pesar de su relevancia para el estudio de cómo los migrantes y sus hogares tratan con diferentes enlaces en distintas localidades, los críticos han cuestionado la novedad de la perspectiva transnacional, la forma en que está definida y la significancia de las prácticas transnacionales para los estudios de migración (Kivisto, 2001; Levitt, Dewind \& Vertovec, 2003, p. 565). Según Olwig y Sørensen (2002, p. 2) "lo difuso de la noción del transnacionalismo puede reducir el campo de investigación a redes y relaciones que involucren el cruce corporal de las fronteras nacionales, porque ésta es la única característica clara y definida del transnacionalismo”. En efecto, se puede argumentar que el transnacionalismo se basa en gran medida en flujos migratorios unidireccionales del Sur hacia los Estados Unidos u otros países “occidentales”. Por tanto, existe el peligro inherente de que la investigación se enfoque exclusivamente en este tipo de "cruces corporales" (Olwig \& Sørensen, 2002, p. 2) en migraciones del sur al norte, dejando de lado otros enlaces entre personas y lugares. La forma en 
que las familias tratan de mejorar su bienestar a través de migraciones estacionales sur-sur, movimientos rurales-urbanos recurrentes y la movilidad diaria, podrían permanecer invisibles.

Para hacer justicia a la diversidad de enlaces, personas y lugares involucrados en la movilidad contemporánea, preferimos hacer uso del concepto de translocalidad (Van Westen \& Zoomers, 2011). La translocalidad abarca diferentes tipos de conexiones a través de la movilidad, intercambio y flujos de información entre diferentes tipos de lugares, por ejemplo rurales y urbanos, tanto en el sur como en el norte. Estas interconexiones afectan el significado y potencial de los lugares para el bienestar y el desarrollo. Para los lugares no necesariamente (o no más prominentemente) involucrados en migraciones sur - norte, el desarrollo translocal puede proveer un marco de trabajo más inclusivo y acertado para analizar la importancia de la movilidad y la interconectividad de los procesos de desarrollo en distintas localidades. Este enfoque en la translocalidad incluye el cruce de fronteras sur - norte, pero no está limitado a este aspecto. En un nivel más amplio, nos anima a basar nuestro análisis de estrategias de vida de los hogares (semi-)rurales en flujos e interconexiones entre personas y lugares.

Estudios de estrategias de vida indican que la diversidad, adaptabilidad y capacidad de recuperación son dimensiones importantes en las estrategias para ganarse la vida (Bebbington, 1999; De Haan \& Zoomers, 2005; Ellis, 2000; Scoones, 2009). Reconociendo la diversidad de estrategias de vida en general y el hecho de que se desarrollan en distintas localidades simultáneamente, se requiere la inclusión explícita de un concepto de movilidad en los estudios sobre medios para ganarse la vida. Olwig y Sørensen (2002, p. 9) argumentan a favor de un concepto de "estrategias de vida móviles" el cual "explora las diversas prácticas involucradas en 'ganarse la vida', así como las relaciones sociales usadas para poder ganársela, en los diferentes contextos en que suceden". Aunque es útil para enfocarse en el uso cotidiano que la gente da a la movilidad y la variedad de formas que ésta puede tomar, nosotros sugerimos que en el análisis de estrategias de vida la noción de movilidad sea incorporada con mucha cautela. El término "estrategias de vida móviles" puede crear la impresión de que los hogares en cuestión están caracterizados por una movilidad constante y libre de obstáculos. Desde esta perspectiva, el concepto ignora a gran escala la importancia del arraigo (Freeman, 2002; Gilbert, 1998) y de los problemas de acceso. La movilidad, como cualquier otro medio de vida, es un recurso mediado por el poder (Sheller \& Urry, 2006, p. 211), que no es disponible para todos (Kothari, 2003). Aún cuando las personas experimentan cierto nivel de movilidad, lo que es probable considerando el continuo flujo de migrantes, no siempre cosechan sus frutos. Esto se vincula con lo que Massey (1993) llama la "geometría del poder" de los flujos contemporáneos. La incorporación de esta noción dentro de nuestro análisis produce un entendimiento más calificado de la relación entre estrategias de vida y movilidad. Por tanto agrega una dimensión importante a nuestro marco teórico para analizar cómo los hogares (semi-)rurales en Nicaragua usan el movimiento como un activo en la construcción de redes y medios de vida a lo largo del tiempo y el espacio. 


\section{Hogares y movilidad en la Nicaragua (semi-)rural}

Nicaragua es el país más rural de Centroamérica y el único en el que el sector agrícola ha venido creciendo (Grigsby \& Pérez, 2007). Distintas zonas rurales en municipios como Matiguás y Muy Muy han experimentado un auge en la producción del ganado lechero debido a una creciente demanda de países extranjeros (como El Salvador y Estados Unidos) y un interés internacional en su industria agrícola (proveniente de compañías como Parmalat). Para ejemplificar, entre 1995 y 2002 los ingresos por productos lácteos se expandieron de U\$4 - 5 millones a U\$40 millones (NitlapanUCA, 2003). A pesar de esta dinámica en la crianza de ganado lechero, la pobreza rural permanece alta y el crecimiento económico esperado no se ha materializado para la mayoría de la población (Bastiaensen \& Marchetti, 2010; Flores \& Delmelle, 2006). Estas dinámicas recientes parecen haber beneficiado a un número limitado de finqueros privilegiados, quienes pueden participar en la lucrativa cadena del ganado lechero. Sin embargo, la mayoría de hogares se encuentran excluidos de estas cadenas o son sólo marginalmente beneficiados y en su mayoría dependen de actividades que proveen un ingreso irregular. Estos factores contribuyen a una alta movilidad humana que también es característica de otras regiones en Nicaragua (Baumeister, 2006; Morales \& Castro, 2002).

Nuestro análisis de las actividades económicas de los informantes confirma que sus opciones en el área investigada están restringidas a actividades agrícolas en pequeña escala y a otras oportunidades de trabajo, inestables y mal pagadas. Los varones entrevistados en las comarcas, por ejemplo, encuentran trabajo por día en haciendas operadas por finqueros, ayudando en actividades como el ordeño de las vacas, arreglando cercas y limpiando la tierra. No obstante, estas actividades dependen grandemente del ciclo agrícola y generalmente son mal pagadas ${ }^{4}$. Otros informantes encuentran trabajo en talleres de carpintería o en los centros de acopio. Sin embargo, estos tipos de trabajo no son necesariamente más estables o mejor pagados que los trabajos por día en una hacienda privada. Los que viven en el pueblo pueden tener más posibilidades en el sector construcción, que tiende a pagar un poco mejor, pero estos trabajos son también muy inestables. Las mujeres entrevistadas en las comarcas también se quejan ante restricciones similares en sus oportunidades económicas. Ocasionalmente realizan costura, lavado y planchado, o ganan algo criando cerdos, vendiendo huevos, pollos o comidas caseras como rosquillas, nacatamales y buñuelos. No obstante, sus oportunidades económicas están en gran medida limitadas por la temporada de cosecha del café, que dura de noviembre a enero, cuando los salarios de cosecha pueden crear un mercado temporal. Sus contrapartes en el pueblo pueden tener mejor acceso a trabajos más estables en pulperías o como trabajadoras domésticas, pero en la práctica nuestros informantes usualmente pasan por alto este tipo de trabajos por razones socioeconómicas. 
Dado que la mayoría de nuestros informantes pertenece a hogares jóvenes, recientemente formados, tienden a depender en gran medida de miembros mayores de su familia para organizar sus estrategias de vida. Aunque algunos, provenientes de las comarcas, a menudo buscan ser independientes a través de la compra de su propia casa, tierra u otros recursos (Sosa \& Steel, 2010). Esto es especialmente difícil de lograr en una localidad caracterizada por inequidad, falta de educación y oportunidades económicas limitadas. Sienten que, para poder ganar el dinero necesario para sobrevivir en las épocas de crisis, mejorar su nivel de vida e independizarse, necesitan irse. En palabras de Sara, "para poder trabajar tenemos que irnos lejos" (entrevista, 17 de febrero de 2010)5. Por tanto no nos sorprende que la mayoría de nuestros informantes hayan experimentado la movilidad. Para los niños en edad escolar viviendo en comunidades sin escuela secundaria, como en el área rural de Muy Muy, las experiencias con la movilidad usualmente inician con viajes a pueblos y ciudades cercanas para continuar estudios. Sin embargo, aún en las comunidades con escuela secundaria hay muchos estudiantes, sobre todo mujeres, que abandonan el hogar para continuar con su educación en otro lugar. La mayoría inicialmente se va a vivir con hermanos u otros parientes en ciudades como Matagalpa y Managua. A cambio de comida y techo, ayudan con las tareas domésticas. Otras buscan empleo como domésticas para asegurarse un lugar donde dormir y un ingreso para pagar su educación. Dado que estos primeros movimientos fuera de su comunidad son generalmente seguidos por varios movimientos de largo y corto plazo, pueden ser considerados como puntos de partida para estrategias de vida caracterizadas por la movilidad y una mayor independencia.

En esta discusión nos enfocamos en tres grupos distintos de personas cuyas estrategias de vida involucran tipos de movilidad que son comunes en esta localidad ${ }^{6}$. El primer grupo consiste en hogares jóvenes cuyas estrategias principales de vida se encuentra afianzadas localmente, pero cuyos adultos varones abandonan la comunidad temporalmente para ganar dinero adicional. En las comarcas, la mayoría de estos hogares subsisten de la agricultura, cultivando frijoles y granos básicos en la parcela de sus padres. Los varones de estos hogares tienden a migrar de noviembre a enero para ganar dinero cosechando café en otros lugares de Nicaragua o Costa Rica. También migran de marzo a mayo, durante la llamada "temporada muerta”, cuando las actividades agrícolas en sus comunidades son poco intensivas, para encontrar trabajo ya sea en el sector agrícola o de la construcción. Las mujeres de estos hogares se quedan en casa encargándose del trabajo doméstico, cuidando de los niños y vigilando los cultivos. En casos de emergencia pueden recibir algún dinero de sus compañeros que se encuentran trabajando en otros lugares, pero en general deben arreglárselas hasta que los hombres regresan. El propósito de la movilidad a corto plazo de los miembros varones de la familia es obtener medios financieros suficientes para mantener sus hogares y ser capaces de realizar pequeñas inversiones en el hogar y en la agricultura. Sin embargo, en la práctica, observamos que el dinero que los

\footnotetext{
5 Todos los nombres han sido cambiados para preservar la confidencialidad.

6 Una tipología de este tipo tiene limitaciones, especialmente porque la movilidad es difícil de categorizar. Sin embargo, distinguir entre los tipos de movilidad más comunes nos ayuda a mejorar nuestro entendimiento en cuanto a su significancia para las estrategias de vida estudiadas en esta investigación.
} 
varones hacen se va en pagar deudas relacionadas con la migración y con varios tipos de micro-crédito en la pulpería y otros lugares. En efecto, muchas familias enfrentan una recurrente falta de dinero y en consecuencia viven de préstamo en préstamo. Varios informantes afirmaron explícitamente que habían dejado sus comunidades en un intento de encontrar formas de pagar deudas pendientes.

Aunque los hogares en el pueblo están involucrados en la agricultura menos directamente, su situación no difiere mucho de la de sus contrapartes en las comarcas, en términos de su dependencia al calendario agrícola, la necesidad de moverse y la inestabilidad de sus ingresos. A lo largo del año muchos miembros varones de estos hogares se van lejos para trabajar en otros lugares de Nicaragua y Costa Rica. Estos varones están más orientados hacia trabajos en el sector de la construcción que los de las comarcas. Solamente toman trabajos agrícolas cuando no hay otros trabajos disponibles. Aparte de eso, sus experiencias de movilidad no difieren fundamentalmente de las de los varones de las comarcas. Aunque no necesitan regresar por sus cultivos y generalmente son capaces de permanecer lejos por períodos más largos, sus ingresos no son necesariamente más estables. En el sector de la construcción los trabajadores generalmente son contratados por la duración de un proyecto, pero en tiempos de crisis económica muchos proyectos son puestos en espera. Esta inseguridad aunada al hecho de que otros trabajos son difíciles de obtener, significa que sus compañeras en casa a veces tienen que pasar meses sin ningún ingreso. Consecuentemente, la situación de las mujeres que se quedan en el pueblo es prácticamente la misma que la de sus contrapartes rurales. Ambas deben sobrevivir la ausencia de sus compañeros con un apoyo económico mínimo. Las mujeres del pueblo a veces realizan actividades agrícolas, pero la mayoría trata de ganar dinero vendiendo productos caseros, distribuyendo productos de belleza, o proveyendo servicios, como lavando, planchando y cosiendo. Sin embargo, el rango más amplio de actividades, productos y clientes en el pueblo no necesariamente significa que hacer frente a la ausencia de sus compañeros sea más fácil. En la práctica, muchas veces rechazan oportunidades de trabajo fuera de casa porque el pago es muy bajo y, en sus palabras, no vale la pena invertir tiempo en ellas. Esta es la razón por la que las familiares de Anabel se molestaron mucho con ella cuando, eventualmente, logró conseguir un trabajo malpagado en un puesto de comida. Según ellas "no deberías de trabajar por casi nada, no deberías de regalar tu trabajo así" (entrevista, 12 de octubre de 2010). Dado que la vida en el pueblo es usualmente cara, también prevalece la costumbre de vivir de préstamo en préstamo, como en las comarcas. 

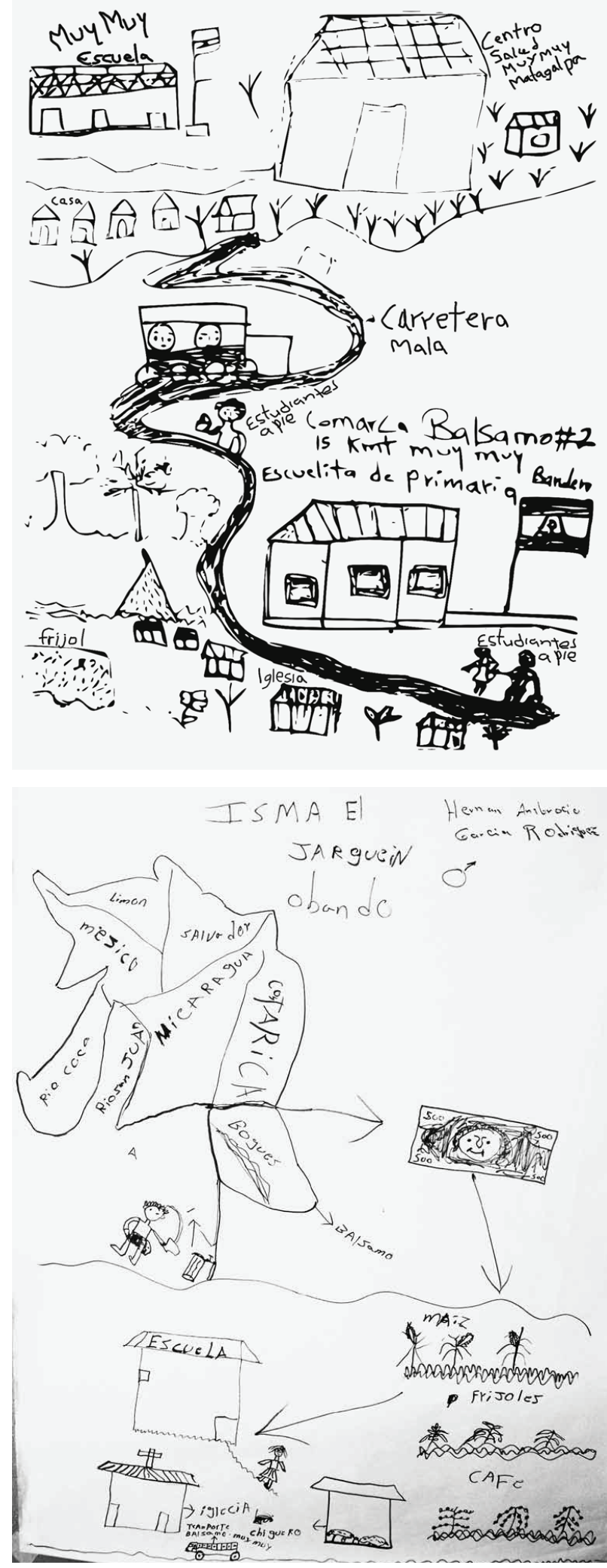

Ilustración 2. Mapa de informantes femeninas

Fuente: Taller sobre la 'Perspectiva de los jóvenes en zonas rurales', organizado en El Bálsamo 2, el 30 de enero de 2010.

Ilustración 3. Mapa de informantes masculinos Fuente: Taller sobre la 'Perspectiva de los jóvenes en zonas rurales', organizado en El Bálsamo, 1 y 3 de febrero de 2010 
El segundo grupo que identificamos se conforma de madres solteras ${ }^{7}$. Como lo ilustra el ejemplo anterior, las mujeres con pareja generalmente se quedan en el hogar aunque su pareja busque trabajo afuera. Pueden haberse ido por razones educativas como mujeres solteras, pero la mayoría de las mujeres abandonan la educación una vez que tienen una pareja estable. Se hacen responsables de sus recién formados hogares y se dedican cada vez más a las actividades domesticas. Además, sus compañeros muchas veces no las dejan trabajar fuera del hogar. Esto en parte se debe a la expectativa de que las mujeres sean amas de casa y también a los antes mencionados, pocos beneficios del trabajo asalariado para las mujeres. Para tener un mejor entendimiento de los patrones de movilidad de hombres y mujeres jóvenes, condujimos un ejercicio de mapeo. El mapa dibujado por los jóvenes de las comarcas confirma la observación de que los hombres tienden a dejar el área de Muy Muy/Matiguás en busca de oportunidades de trabajo, pero que el movimiento de las mujeres está usualmente restringido a la comunidad, donde la mayoría de sus actividades toma lugar. Las mujeres algunas veces van a la ciudad a comprar, estudiar o visitar al médico, pero mientras más lejos viven, menos frecuentes son estos movimientos. Los mapas dibujados por las mujeres del pueblo muestran que sus movimientos están más restringidos al pueblo y las comarcas aledañas, usualmente para visitar parientes. Sólo en casos de emergencia u otro tipo de eventos mayores es probable que viajen a ciudades vecinas.

Sin embargo, este patrón parece cambiar tan pronto las mujeres se convierten en madres solteras y tienen que enfrentar el reto de criar sus hijos solas. La mayoría de estas mujeres no ve suficientes oportunidades para ganarse la vida en su comunidad y, en consecuencia, frecuentemente buscan trabajo doméstico pagado en las ciudades de Nicaragua y Costa Rica. En contraste con el primer grupo, estas madres solteras encuentran relativamente fácil el obtener un trabajo durante todo el año en el sector doméstico. Sin embargo, como es casi imposible encontrar trabajo doméstico pagado estando acompañada de niños, la mayoría de las madres solteras dejan a sus hijos en la comunidad con sus padres, hermanas u otros parientes. El dinero que las madres ganan en otro lugar es utilizado en la educación de sus hijos y los gastos diarios de las familias en la comunidad. La duración de la ausencia de la madre depende de las dinámicas de las oportunidades laborales urbanas así como de factores relacionados con la familia. Por ejemplo, Mira es una madre soltera de veinticinco años que usualmente trabaja en Managua mientras deja a su hijo de tres años con su madre en la comarca. Sin embargo, al momento de la entrevista se encontraba viviendo nuevamente en la comunidad porque su hijo se había enfermado. Este caso y otros parecidos indican que la dinámica del cuido de los niños es una razón importante para que las mujeres regresen a sus hogares hasta que puedan hacerse nuevos arreglos (Winters, 2010). En contraste con el primer grupo, el momento en el que dejan la comunidad no depende del ciclo agrícola. Las estrategias de vida de estas mujeres generalmente no están explícitamente orientadas hacia la agricultura, por lo que pueden irse cuando lo estimen conveniente. La disponibilidad permanente de

7 En este artículo se define a una madre soltera como una mujer que cría a su hijo sola. Esto no necesariamente indica que no recibe apoyo de su(s) ex compañero(s). 
trabajos para mujeres en la ciudad hace relativamente fácil para ellas el encontrar un nuevo empleo. Consecuentemente, su posición en el mercado de trabajo es menos precaria que la de los hombres pertenecientes al primer grupo.

Finalmente identificamos un pequeño, pero digno de mencionar, tercer grupo que consiste en parejas que viajan a Costa Rica con la esperanza de ganar suficiente dinero para iniciar una vida independiente en su comunidad de origen. El que lleven o no a sus hijos con ellos depende de muchos factores. Más importante aún, como en el caso de las madres solteras, las oportunidades que generan ingresos en el exterior se ven limitadas para las personas acompañadas por niños. Eliana y Julio, por ejemplo, decidieron llevar a sus hijos a Costa Rica con ellos luego de años trabajando allá sin ellos y habiéndolos extrañado demasiado. Sin embargo, no mucho tiempo después los enviaron de regreso a Muy Muy con unos familiares porque no podían permitirse tenerlos con ellos en Costa Rica: no sólo sus gastos diarios incrementaron significativamente, sino que la capacidad de trabajo de Eliana disminuyó a causa del peso agregado por las labores domésticas. Aparentemente sólo un pequeño grupo de parejas bien establecidas puede permitirse vivir en Costa Rica con sus hijos. Los otros trabajan en el extranjero y dejan a sus hijos en casa. De este modo incrementan sus oportunidades de acumular activos, mejorando sus estrategias de vida y ganando independencia.

Habiendo identificado estos diferentes grupos y sus principales aspiraciones y objetivos, en la próxima sección dirigimos nuestra atención a las estrategias utilizadas por nuestros informantes para alcanzar sus objetivos y las implicaciones para su bienestar y la comunidad. Más específicamente, consideramos qué conexiones se establecen entre las personas y los lugares.

\section{La movilidad como un recurso negociado}

Zoomers y Van Westen (2011) definen el concepto de "corredores de desarrollo" como un conjunto interconectado de personas y lugares en el que toman lugar intercambios y movimientos. El concepto ofrece un marco útil para tener un mejor entendimiento de las implicaciones de la movilidad para los procesos de desarrollo en Matiguás y Muy Muy. Sugiere que el bienestar depende de las conexiones entre personas y lugares, más que de los aspectos "estáticos" tales como los lugares desde y hacia los cuales las personas se movilizan, por cuánto tiempo y por qué. Por tanto, aplicamos el concepto en la exploración de cómo la movilidad establece conexiones y cómo éstas se relacionan a los procesos de desarrollo a nivel de los hogares y de la comunidad.

Primero analizamos el caso de los hogares cuyos miembros varones abandonan la comunidad temporalmente. Como se indicó previamente, la mayoría de nuestras informantes son excluidas de este tipo de movilidad debido a sus responsabilidades familiares. Sin embargo, eso no significa que ellas no estén involucradas de otra forma con la movilidad. Partiendo de la noción de que mejorar el bienestar de un hogar es un esfuerzo mutuo (Pribilsky, 2007), vemos que las mujeres generalmente se benefician de los mayores flujos de efectivos de sus parejas, la capacidad de pagar préstamos e invertir en recursos. Adicionalmente, las mujeres en el pueblo a menudo indican que disfrutan el vivir solas con sus hijos. Como María explicó, 
cuando su pareja está en casa, él demanda toda su atención, discuten sobre los niños y, al no estar acostumbrados a su mutua presencia, solamente consiguen alterarse (entrevista, 17 de julio de 2009). Aunque ella disfruta de estar un par de días juntos, generalmente encuentra más llevadero el tener a su pareja lejos. Sin embargo, las entrevistadas también enfatizan ciertos inconvenientes al tener a sus parejas lejos. En las comarcas las mujeres usualmente perciben la ausencia de sus parejas como una carga pesada: las deja con mayores responsabilidades, como atender los cultivos, y a menudo las hace sentirse solas, especialmente cuando viven en un relativo aislamiento. Adicionalmente, tal como se mencionó antes, las mujeres en las comarcas, así como en el pueblo, deben acudir a sus propias estrategias para arreglárselas mientras sus esposos están lejos y temporalmente incapacitados para enviar dinero. En este sentido las mujeres que se quedan en las comarcas y en el pueblo están directa e indirectamente involucradas con los costos y beneficios de los movimientos de sus parejas.

A nivel de la comunidad, las oportunidades de desarrollo local están muy relacionadas con las dinámicas de la movilidad. Es aparente que la creación de ciertas oportunidades económicas para la comunidad tiene más probabilidad de aparecer cuando los migrantes regresan. Esto es más notable en las comarcas, donde las personas tienen en cuenta la temporada agrícola. Los migrantes de temporada tienden a estar en casa cuando los cultivos necesitan cosecharse y lejos cuando no se necesita realizar un trabajo pesado. En otras palabras, regresan a casa para explotar oportunidades agrícolas. Sin embargo, al mismo tiempo, su regreso genera empleos en otros sectores. Por ejemplo, para los carpinteros, diciembre, enero y mayo tienden a ser meses financieramente gratificantes, ya que este es el tiempo cuando los migrantes se encuentran en casa y por tanto son capaces de gastar sus ganancias en muebles (entrevista con Jonás, 15 de febrero de 2010). En el pueblo las oportunidades económicas parecen estar distribuidas más uniformemente a lo largo del año y dependen mucho menos del calendario agrícola. Sin embargo, aún aquí, los artesanos y negocios locales son afectados por la irregularidad del ingreso en los hogares, ya que generalmente no reciben sueldo hasta que el dinero es enviado o traído por los migrantes mismos. Este efecto es sentido con mayor fuerza por los dueños de pulperías, prestamistas y vendedores de materiales de construcción.

La conexión cercana entre la economía local y las dinámicas de la migración también puede ilustrarse con el caso de la crisis económica global del 2008. La recesión y el consiguiente declive en las oportunidades de empleo, especialmente en el sector de la construcción, significaron una pérdida de trabajo para muchos migrantes. Esto tuvo implicaciones directas tales como visibles disminuciones en los flujos de dinero e inversiones locales, incremento en préstamos y más faltas de pago. Esto sugiere que puede valer la pena examinar oportunidades y restricciones de desarrollo en el contexto de las llamadas "cadenas de desarrollo", las que toman debidamente en cuenta las interconexiones de las dinámicas socioeconómicas en diferentes localidades (ver Zoomers \& Van Westen, 2011).

El análisis anterior muestra que no sólo hay distintos tipos de actores involucrados en la movilidad, pero que también los otros actores están directamente vinculados con los costos y beneficios de tal movilidad. Como otros han discutido (Kothari, 2003; Levitt \& Jaworsky, 2007; Vertovec, 2004), la movilidad tiene 
el potencial de cambiar vidas más allá de las de los migrantes y sus hogares. Adicionalmente, el énfasis de nuestros informantes en las oportunidades de desarrollo en sus comunidades parece sugerir que la mayoría aún aspira a un futuro en su localidad. Cuando es posible, invierten en activos como vivienda y educación. En este sentido, no utilizan la movilidad para empezar de nuevo, más bien lo hacen para incrementar su potencial socioeconómico dentro de su comunidad de origen (ver también Sørensen \& Olwig, 2002; Thieme, 2008). Es la combinación de las implicaciones de largo alcance que tiene la movilidad para los diferentes actores y la continua importancia de la comunidad de origen, lo que resulta en claros corredores de desarrollo. En este caso, los corredores pueden ser caracterizados como fluidos en el sentido de que los movimientos involucrados, aunque de cierto modo predecibles, no tienden a generar ni ingresos más regulares para el hogar, ni oportunidades económicas estables para la economía local. En cambio, observamos un corredor de desarrollo que está muy orientado hacia la subsistencia y el pago de deudas, y en hacer inversiones modestas para el futuro.

En el caso del segundo grupo, las madres solteras, el corredor de desarrollo que se establece por medio de su uso particular de la movilidad luce ligeramente diferente. En comparación con el primer grupo, estas mujeres experimentan menos fluctuaciones en las oportunidades laborales y tienen más probabilidad de encontrar un trabajo en caso de perder el actual. Por tanto, su ingreso es usualmente más estable y el flujo de remesas es más permanente a lo largo del año. Estas remesas son generalmente utilizadas para pagar los préstamos en que incurren quienes cuidan a sus hijos con los negocios locales, como pulperías y farmacias. Sin embargo observamos una notable diferencia en el comportamiento de las inversiones de estas madres solteras y las del primer grupo. Mientras los hogares en los que el varón abandona temporalmente la comunidad están inclinados a combinar gastos en educación para los niños con la adquisición de activos como tierra y vivienda, el último tipo de gasto parece tener menor prioridad para las madres solteras. Usualmente no aspiran a vivir independiente de sus padres, ya que dependen mucho de la red de cuido en casa. Cabe agregar que generalmente es considerado inapropiado que las mujeres vivan solas, sin un compañero o familia extendida. Sin la presión inmediata de adquirir tierras y vivienda, parecen tener un mayor rango de opciones para invertir continuamente en la educación de sus hijos. Además de ser más estable, el corredor establecido a través de la movilidad de las madres solteras está también más explícitamente orientado hacia el mejoramiento de la educación de sus hijos.

Es importante enfatizar que en este segundo caso, también, las opciones de movimiento y la manera de moverse están en dependencia de la participación de otros actores. Para aprovechar al máximo las oportunidades de trabajo que se les presentan y para mejorar su calidad de vida, las madres solteras recurren al apoyo de sus padres y otros familiares para cuidar a sus hijos. Como Leinaweaver (2008) observó en el caso de "circulación de niños" en Perú, la práctica de dejar a los hijos en casa de algún pariente es muy importante para los proyectos familiares de seguir adelante. Esto plantea preguntas sobre las implicaciones de la red de cuido resultante para las estrategias de vida de quienes se quedan (ver Schmalzbauer, 2010, pp. 1863-4). En las comarcas observamos que, generalmente hablando, los abuelos 
se vuelven cuidadores cuando sus hijas se van y dejan a sus niños atrás. En tales casos, el apoyo de la madre soltera es un ingreso importante para el sustento del hogar extendido. En el pueblo la mayoría de las abuelas no está disponible para cuidar de sus nietos a tiempo completo, ya que la mayor parte del día realizan actividades productivas fuera del hogar. En este caso, la responsabilidad del cuido de los niños recae sobre la pariente más cercana, usualmente una hermana de la madre soltera. Esta responsabilidad disminuye significativamente las oportunidades de esta hermana para poder obtener oportunidades de vida en otro lugar. Aunque, como los abuelos, generalmente se beneficia del flujo de dinero que la madre soltera genera, el hecho de que otros se muevan limita su propia movilidad y tiende a poner en pausa sus propios planes para obtener una estrategia de vida independiente. Este acceso desigual es un ejemplo concreto de la "geometría del poder" de la movilidad (Massey, 1993), a través de la cual los movimientos de algunas personas pueden, de hecho, disminuir las posibilidades de otras. Nuevamente ilustra que los movimientos de los actores definidos en la sección previa están íntimamente relacionados con la falta de movimiento de quienes se quedan.

Finalmente, como un tercer grupo, identificamos a parejas que se van con o sin sus hijos con el propósito de acumular una cantidad de capital suficiente para poder invertir en tierra, vivienda y, si es posible, un pequeño negocio en sus comunidades de origen. En los casos en que estas parejas han dejado a sus hijos con parientes, el consiguiente corredor de desarrollo no difiere fundamentalmente del de las madres solteras. En ambos casos se envía dinero a la familia extendida en casa para gastos diarios y la educación de los niños que se quedaron. Sin embargo, las parejas pueden tener más éxito en su objetivo de construir una vida independiente dentro de su propia comunidad por el simple hecho de que ambos compañeros tienen el propósito de ganar dinero afuera. En los casos en que la pareja lleva a sus hijos consigo, las características del corredor de desarrollo cambian significativamente porque, como es usual, el flujo de efectivo hacia la comunidad no es continuo. Las familias mantienen enlaces con su comunidad de origen, pero sólo envían dinero a sus parientes en ocasiones, como un regalo o en casos de emergencia. Las ganancias son generalmente utilizadas para los gastos de la residencia temporal de la familia o apartados para ser invertidos una vez regresen a la comunidad. En este sentido, su movilidad da forma a un corredor de desarrollo "en espera". Sólo cuando la familia regresa a su comunidad de origen para construir un mejor futuro es que crean oportunidades económicas tangibles, al invertir en tierra, vivienda y otros. Mientras se encuentren lejos, las familias generalmente mantienen otros enlaces más allá de los monetarios. A través de la comunicación, intercambian ideas y nutren la esperanza del retorno. Mientras el último caso sugiere que los corredores de desarrollo no están restringidos a los flujos de capital y que también se involucran otros aspectos, es claro que el dinero es un catalizador importante para un corredor de desarrollo. Al mismo tiempo, el caso también corrobora nuestro argumento de que los corredores toman forma por el hecho de que ambos, los que se van y los que se quedan, están involucrados con la movilidad. Cuando las parejas se vuelven (temporalmente) menos dependientes de los que se quedan y se distancian más de los procesos de desarrollo de su comunidad de origen, el corredor de desarrollo se torna menos explícito. 


\section{Conclusión}

El punto de inicio de esta investigación fue la observación de que los discursos locales sobre movilidad social están fuertemente asociados con la idea de movilidad espacial. Es decir, el deseo de seguir adelante anima a las personas a irse del área de Matiguás/Muy Muy. A menudo, distintos miembros del hogar han migrado en varias ocasiones, a diferentes lugares y por diferentes períodos. Al momento de escribir este texto, algunos habrán regresado mientras que otros pueden haberse movido a otra localidad. Dado que la movilidad es ubicua y casi inevitable e impregna las estrategias de vida de las personas de maneras dinámicas e impredecibles, sigue siendo un reto interpretar el nexo entre la movilidad y el desarrollo. Sin embargo, en este artículo hemos contribuido a un mejor entendimiento de estos nexos al incorporar en el análisis el concepto de desarrollo translocal. Esto a su vez nos permite identificar corredores de desarrollo para analizar las implicaciones directas e indirectas de la diversidad de movimientos que son parte de las estrategias de vida de los hogares (semi-)rurales en Nicaragua.

Las maneras en que estos corredores toman forma ejemplifica la complejidad de la relación entre movilidad y desarrollo. Observamos, por ejemplo, que los objetivos al salir son un componente importante de la forma en que estos corredores se materializan. Schapendonk (2011) argumenta que los estudios de migración tienden a sobre enfatizar las intenciones y racionalidades de los migrantes, mientras que debiesen enfocarse en los flujos, interdependencias y oportunidades múltiples. Si bien concordamos con este razonamiento, nuestros hallazgos indican que, a pesar de las oportunidades, los objetivos y logros fluctuantes, nuestros informantes trabajan con su vista puesta en metas claras, aunque sencillas. Primeramente, se mueven para ganar dinero y pagar préstamos, para la educación de sus hijos y para acumular suficientes activos que les permitan ser independientes una vez regresen a casa. Aunque no encuentren muchas oportunidades socio-económicas en sus comunidades, sí aspiran a cumplir un futuro en ella. La noción de mejorar la propia vida en la comunidad implica que el enlace entre personas y lugares finalmente da forma a los corredores de desarrollo.

Otro componente importante que da forma a los corredores de desarrollo son las varias maneras en que distintos actores - los que se van y los que se quedan - se involucran con la movilidad. A través de enlaces entre personas y lugares, la movilidad da forma a las estrategias de vida más allá de los grupos identificados. Esto se ve en el hecho de que quienes no están dispuestos o no pueden irse pueden aún estar involucrados en la movilidad. También es aparente en la estrategia de vida explícita de reservar la movilidad para algunos, mientras se le niega a otros, de acuerdo a las circunstancias. Por tanto, la movilidad puede ser considerada como un recurso negociable, y la manera en que es manejada, así como la forma en que sus costos y beneficios son compartidos, resulta ser instrumental para el bienestar de los hogares y las oportunidades comunales.

Estas observaciones claramente llaman a una visión más calificada y matizada de la movilidad. Partiendo de la noción de Massey (1993) de una "geometría del poder", proponemos el concepto de un espectro de la movilidad a la no movilidad 
por medio de diferentes arenas de poder, tales como acceso, responsabilidades y dinámicas contextuales. Esta interpretación más amplia de la movilidad identifica a las personas y lugares involucrados y los mecanismos responsables de la construcción de corredores de desarrollo entre ellos. Esto nos ha permitido interpretar diferentes formas en que los corredores pueden asumir y arrojar luz sobre la importancia del desarrollo translocal a nivel de hogares y comunidades en la Nicaragua (semi-)rural. Por ejemplo, los corredores más fluctuantes, formados por hombres, dan lugar a diferentes tipos de procesos de desarrollo translocal que los corredores "en espera", formados por las parejas. Estamos conscientes de que la comprensión de tales corredores de desarrollo sería significativamente mejorada por la incorporación de observaciones del otro lado (o lados) de estos corredores, por ejemplo, los diversos lugares en Nicaragua, Costa Rica y demás. No obstante, la noción del espectro de movilidad que proponemos ha contribuido sustancialmente a nuestras percepciones de los nexos entre movilidad y desarrollo, y provee un valioso punto de partida para un enfoque más comprensivo.

\section{Agradecimiento}

Este trabajo de investigación fue financiado por el Consejo de Universidades Flamencas VLIR-USOS y el fondo de Investigación del Instituto de Política y Gestión y Desarrollo (IOB) de la Universidad de Amberes. Agradecemos a Johan Bastiaensen y Pierre Merlet por el proceso de coordinación de este número especial de Encuentro. También expresamos nuestra gratitud a los informantes de Matiguás y Muy Muy, quienes amablemente dedicaron su tiempo a nuestra investigación.

\section{Referencias bibliográficas}

Bastiaensen, J. \& Marchetti, P. (2010). Microfinanzas rurales y cadenas de valor agropecuarias. IOB Working Paper, (02), Amberes: Instituto de Política y Gestión y Desarrollo (IOB).

Baumeister, E. (2006). Migración internacional y desarrollo en Nicaragua. Serie población y Desarrollo, (67). Santiago de Chile: Naciones Unidas.

Bebbington, A. (1999). Capitals and capabilities: a framework for analyzing peasant viability, rural livelihoods and poverty. World Development, (27), 2021-2044.

Bebbington, A. J. \& Batterbury, S. P. J. (2001). Transnational livelihoods and landscapes: political ecologies of globalization. Ecumene, (8), 369-380.

Brandao, F. \& Zoomers, A. (2010), Solade di nhos terra: the development potential of return migration to Cape Verde. International Development Planning Review, (33), 267-289.

Collier, J. \& Collier, M. (1996). Visual Anthropology. Photography as a Research Method. Albuquerque: University of New Mexico Press.

Collins, D., Mordoch, J., Rutherford, S. \& Ruthven, O. (2009). Portfolios of the Poor. How the World's Poor Live on $\$ 2$ a Day. Princeton: Princeton University Press.

Cresswell, T. (2006). On the Move. Mobility in the Modern Western World. London: Routledge. 
De Haan, L. (1999). Livelihoods and poverty: the role of migration - a critical review of the migration literature. Journal of Development Studies, (36), 1-47.

De Haan, L. (2006). Migration in the development studies literature. Has it come out of its marginality?. Research Paper, (19). Helsinki: UNU World Institute for Development Economics Research (UNU-WIDER).

De Haan, L. \& Zoomers, A. (2003). Development geography at the crossroads of livelihood and globalization. Journal of Economic and Social Geography (TESG), (94), 350-362.

De Haan, L. \& Zoomers, A. (2005). Exploring the frontier of livelihoods research. Development and Change, (36), 27-47.

De Haas, H. (2005). International migration, remittances and development: myths and facts. Third World Quarterly, (26), 1269-1284.

De Haas, H. \& Rodríguez, F. (2010). Mobility and human development: introduction. Journal of Human Development and Capabilities, (11), 177-184.

Duany, J. (2002). Mobile livelihoods: the sociocultural practices of circular migrants between Puerto Rico and the United States. International Migration Review, (36), 355-388.

Ellis, F. (2000). Rural Livelihoods and Diversity in Developing Countries. Oxford: Oxford University Press.

Flores, S. \& Delmelle, G. (2006). Detailed market chain analysis for smallholders affected by the CAFTA Agreement in Nicaragua, Managua. Informe para IFPRI. Managua: Nitlapan.

Freeman, C. (2002). Mobility, rootedness, and the Caribbean higgler: production, consumption and transnational livelihoods. En N. Sørensen \& K. Olwig (eds.). Work and Migration: Life and Livelihoods in a Globalizing World. (pp. 6181). London: Routledge.

Gilbert, M. R. (1998). "Race,” space, and power: the survival strategies of working poor Women. Annals of the Association of American Geographers, (88), 595-621.

Glick Schiller, N., Basch, L. \& Blanc-Szanton, C. (1992). Transnationalism: a new analytic framework for understanding migration. En S. Vertovec \& R. Cohen (eds.). Migration, Diasporas and Transnationalism. (pp. 26-49). Cheltenham: Edward Elgar.

Grigsby, A. \& Pérez, E. (2007). Structural Implications of Economic Liberalization on Agriculture and Rural Development in Nicaragua. First Phase: National Synthesis ES Second phase: Procesos de Diferenciación de la Población y la Economía Rural. Managua: Nitlapan.

Kivisto, P. (2001). Theorizing transnational immigration: a critical review of current efforts. Ethnic and Racial Studies, (24), 549-577.

Kothari, U. (2003). Staying put and staying poor?. Journal of International Development, (15), 645-657.

Leinaweaver, J. (2008). Improving oneself: young people getting ahead in the Peruvian Andes. Latin American Perspectives, (35), 60-78.

Levitt, P., Dewind, J. \& Vertovec, S. (2003). International perspectives on transnational migration: an introduction. International Migration Review, (37), $565-575$.

Levitt, P. \& Jaworsky, B. N. (2007). Transnational migration studies: past 
developments and future trends. Annual Review of Sociology, (33), 129-156.

Massey, D. (1991). A global sense of place. Marxism Today, July, 24-29.

Massey, D. (1993). Power-geometry and a progressive sense of place. En J. Bird, B. Curtis, T. Putnam \& G. Robertson (eds.). Mapping the Futures: Local Cultures, Global Change. (pp. 59-69). London: Routledge.

Morales, A. \& Castro, C. (2002). Redes Transfronterizas. Sociedad, Empleo y Migración entre Nicaragua y Costa Rica. San José, Costa Rica: Facultad Latinoamericana de Ciencias Sociales (FLACSO).

NITLAPAN-UCA (Universidad Centroamericana). (2003). Cadena de lácteos: la inserción de las mujeres a la economía globalizada: Estudio de caso Municipios de Boaco y Santo Tomás en Nicaragua. Managua: OIT/UNIFEM/PNUD.

Olwig, K. \& Sørensen, N. (2002). Mobile livelihoods: making a living in the world. En N.

Sørensen \& K. Olwig (eds.). Work and Migration: Life and Livelihoods in a Globalizing World. (pp. 1-21). London: Routledge.

Paerregaard, K. (1998). The dark side of the moon: conceptual and methodological problems in studying rural and urban worlds in Peru. American Anthropologist, (100), 397-408.

Pribilsky, J. (2007). La Chulla Vida: Gender, Migration, and the Family in Andean Ecuador and New York City. New York: Syracuse University Press.

Schapendonk, J. (2011). Turbulent trajectories: Sub-Saharan African migrants heading north. Disertación de doctorado no publicada. Nijmegen, Radboud University Nijmegen, Faculteit der Management Wetenschappen.

Schmalzbauer, L. (2010). Disruptions, dislocations, and inequalities: transnational Latino/a families surviving the global economy. The North Carolina Law Review, (88), 1857-1872.

Scoones, I. (2009). Livelihoods perspectives and rural development. Journal of Peasant Studies, (36), 171-196.

Sheller, M. \& Urry, J. (2006). The new mobilities paradigm. Environment and Planning A, (38), 207-226.

Sørensen, N. \& Olwig, K. (2002). Work and Migration: Life and Livelihoods in a Globalizing World. London: Routledge.

Sosa, C. \& Steel, G. (2010). Rutas de desarrollo: perspectivas y estrategias de vida de los jóvenes rurales en Matiguás y Muy Muy. Informe para el VLIR. Managua: Nitlapan.

Steel, G. \& Zoomers, A. (2009). Social mobility in the Central Andes of Peru and Bolivia: towards a more dynamic and multi-local vision of poverty alleviation. International Development Planning Review, (31), 377-396.

The economist. (2010). Full converter. Recuperado el 10 de enero de 2011, de http:// www.economist.com/markets/currency/fullconverter.cfm

Thieme, S. (2008). Living in transition: how Kyrgyz women juggle their different roles in a multi-local setting. Gender Technology and Development, (12), 3253454.

United Nations Development Programme. (2009). Overcoming Barriers: Human Mobility and Development (Human Development Report). New York: Palgrave Macmillan. 
Urry, J. (2007). Mobilities. Cambridge: Polity Press.

Vertovec, S. (2004). Trends and impacts of migrant transnationalism. Working Paper, (3). Oxford: University of Oxford, Centre on Migration, Policy and Society.

Waldinger, R. \& Fitzgerald, D. (2004). Transnationalism in question. The American Journal of Sociology, (109), 1177-1195.

Willis, K. (2010). Introduction: migration, mobility and development. International Development Planning Review, (32), i-xiii.

Winters, N. (2010). Translocal care work among migrant families in Muy Muy, Nicaragua. Documento presentado en el Encuentro de la American Anthropological Association, New Orleans, 17-21 de noviembre de 2010 (paper).

Zoomers, A. \& Van Westen, G. (2011). Introduction: translocal development, development corridors and development chains. International Development Planning Review, (33), 377-88. 